\title{
Research Article \\ Effect of Mechanism Error on Input Torque of Scroll Compressor
}

\author{
Man Zhao, Shurong Yu, Chao Li, and Yang Yu \\ College of Petrochemical Technology, Lanzhou University of Technology, Lanzhou 730050, China \\ Correspondence should be addressed to Man Zhao; zhao_man79@163.com
}

Received 29 July 2013; Accepted 13 October 2013

Academic Editor: Ryoichi Samuel Amano

Copyright (C) 2013 Man Zhao et al. This is an open access article distributed under the Creative Commons Attribution License, which permits unrestricted use, distribution, and reproduction in any medium, provided the original work is properly cited.

\begin{abstract}
Based on the fundamental principle of plane four-bar mechanism, the force on the equivalent parallel four-bar mechanism was analyzed for scroll compressor with mini-crank antirotation, and the formula of input torque was proposed. The change of input torque caused by the mechanism size error was analyzed and verified with an example. The calculation results show that the mechanism size error will cause large fluctuation in input torque at the drive rod and connecting rod collinear and the fluctuation extreme value increases with rotational speed. Decreasing of the crankshaft eccentricity errors is helpful for reducing the effects of dimension error on input torque but will increase the friction loss of orbiting and fixed scroll wrap. The influence of size error should be considered in design in order to select suitable machining accuracy and reduce the adverse effect caused by size error.
\end{abstract}

\section{Introduction}

With the needs of clean and oil-free pollution compressed air in food, medicine, fuel cells, and other industries, the investigation of oil-free scroll compressor research has been one of hot topics in scroll compressors. The mini-crank antirotation mechanism is often used to achieve the orbiting scroll work under the oil-free lubrication conditions. The movement relationship of mini-crank, orbiting scroll, crankshaft, and the bracket body can be represented by planar four-bar mechanism [1], and the ideal working should be planar parallel four-bar mechanism. But in fact, machining error and running wear are inevitable, such as the orbiting scroll deviating from the ideal state, resulting in excessive friction wear or large tangential leaking [2], also causing the movable linkages uneven movement. The research of the size error on the four-bar mechanism critical rod's uneven movement and the change of input torque can provide some theoretical foundation for machining accuracy and scroll compressor reliable operation.

\section{Four-Bar Linkage Mechanism}

The structure of scroll compressor with mini-crank antirotation is shown in Figure 1. The mini-crank is respectively connected with the orbiting scroll and the bracket body through the bearing. In order to ensure the orbiting scroll's revolving translational motion, the gyration radius of minicrank and eccentric crankshaft should be in the same size; that is, the plane equivalent four-bar mechanism of scroll compressor, shown in Figure 2, should be the parallel fourbar mechanism.

Figure 2 illustrates the four-bar mechanism in the scroll compressor work, $l_{1}$ is equivalent to the drive crankshaft, $l_{2}$ is equivalent to the orbiting scroll, $l_{3}$ is equivalent to the mini-crank, and $l_{4}$ is equivalent to the bracket body. In ideal working conditions: $l_{1}=l_{3}, l_{2}=l_{4}$.

2.1. Geometrical Theory. Cartesian coordinate system is shown in Figure 2; the vector equation is

$$
\begin{gathered}
l_{1} \cos \theta_{1}+l_{2} \cos \theta_{2}=l_{3} \cos \theta_{3}+l_{4}, \\
l_{1} \sin \theta_{1}+l_{2} \sin \theta_{2}=l_{3} \sin \theta_{3} .
\end{gathered}
$$

Equation (1) can be written as

$$
\begin{aligned}
& A \cos \theta_{2}+B \sin \theta_{2}=C, \\
& A \cos \theta_{3}+B \sin \theta_{3}=D,
\end{aligned}
$$




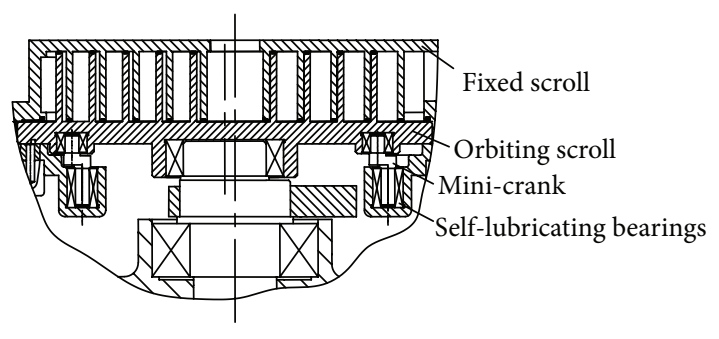

Figure 1: Configuration diagram of scroll compressor.

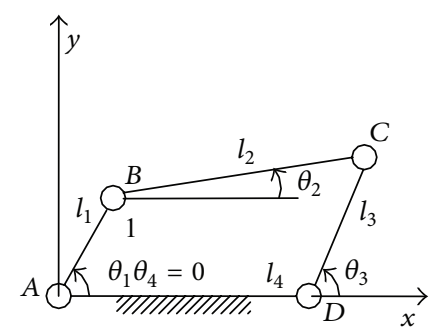

Figure 2: Four-bar mechanism.

where

$$
\begin{gathered}
A=l_{4}-l_{1} \cos \theta_{1}, \\
B=-l_{1} \sin \theta_{1}, \\
C=\frac{1}{2 l_{2}}\left(l_{2}^{2}-l_{3}^{2}+A^{2}+B^{2}\right), \\
D=\frac{1}{2 l_{3}}\left(l_{2}^{2}-l_{3}^{2}-A^{2}-B^{2}\right) .
\end{gathered}
$$

According to (2) and (3), the output angles $\theta_{2}$ and $\theta_{3}$ of four-bar mechanism can be expressed as

$$
\begin{aligned}
& \theta_{2}=2 \arctan \frac{B-M \sqrt{A^{2}+B^{2}-C^{2}}}{A+C}, \\
& \theta_{3}=2 \arctan \frac{B-M \sqrt{A^{2}+B^{2}-D^{2}}}{A+D} .
\end{aligned}
$$

The output angles $\theta_{2}$ and $\theta_{3}$ of the mechanism have two solutions, and the exact value can be determined according to the mechanism's initial installation and the continuity of the movement. When the hinge point $B, C$, and $D$ are arranged clockwise, $M$ will take the positive sign; when the hinge points $B, C$, and $D$ are arranged anticlockwise, $M$ has a negative sign.

Taking first derivative and second derivative of vector equation (1), the angular velocity and angular acceleration of rod 2 and rod 3 can be obtained:

$$
\dot{\theta}_{2}=\frac{l_{1} \sin \left(\theta_{1}-\theta_{3}\right)}{l_{2} \sin \left(\theta_{3}-\theta_{2}\right)} \dot{\theta}_{1},
$$

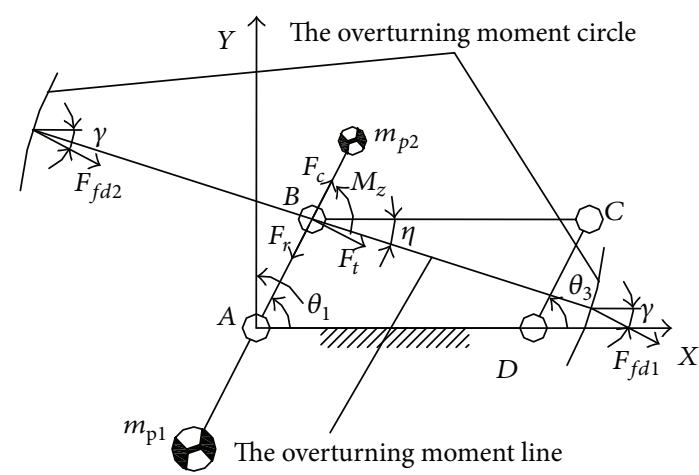

FIgURE 3: Schematic diagram of external loading.

$$
\begin{gathered}
\dot{\theta}_{3}=\frac{l_{1} \sin \left(\theta_{1}-\theta_{2}\right)}{l_{3} \sin \left(\theta_{3}-\theta_{2}\right)} \dot{\theta}_{1}, \\
\ddot{\theta}_{2}=\frac{-\dot{\theta}_{1}^{2} l_{1} \cos \left(\theta_{1}-\theta_{3}\right)-\dot{\theta}_{2}^{2} l_{2} \cos \left(\theta_{2}-\theta_{3}\right)+\dot{\theta}_{3}^{2} l_{3}}{l_{2} \sin \left(\theta_{2}-\theta_{3}\right)}, \\
\ddot{\theta}_{3}=\frac{\dot{\theta}_{1}^{2} l_{1} \cos \left(\theta_{1}-\theta_{2}\right)+\dot{\theta}_{2}^{2} l_{2}-\dot{\theta}_{3}^{2} l_{3} \cos \left(\theta_{3}-\theta_{2}\right)}{l_{3} \sin \left(\theta_{3}-\theta_{2}\right)} .
\end{gathered}
$$

For the parallel four-bar mechanism, when the drive rod is running at a constant angular velocity, the following results can be obtained:

$$
\dot{\theta}_{2}=0, \quad \ddot{\theta}_{2}=0, \quad \dot{\theta}_{3}=\dot{\theta}_{1}, \quad \ddot{\theta}_{3}=0 .
$$

2.2. Mechanism Motion Analysis. By the scroll compressor structure, the active bars centroid coordinates are

$$
\begin{gathered}
x_{S 2}=x_{S 1}=l_{1} \cos \theta_{1}, \\
y_{S 2}=y_{S 1}=l_{1} \sin \theta_{1}, \\
x_{S 3}=l_{4}+l_{3} \cos \theta_{3}, \\
y_{S 3}=l_{3} \sin \theta_{3} .
\end{gathered}
$$

Respectively, by seeking the first derivative and second derivative of (8) and (9), the angular velocity and angular acceleration of the movable rods can be obtained.

\section{Mechanical Models}

While four-bar mechanism is working, in addition to the input torque, the force, and moment acting on mechanism are mainly three types: each movable rod gravity, inertia force, inertia moment; external forces and moment; hinge friction and friction moment.

For the equivalent four-bar mechanism of scroll compressor, external loading in radial plane is shown in Figure 3; tangential gas force $F_{t}$, radial gas force $F_{r}$, rotation torque $M_{z}$, $F_{f d 1}, F_{f d 2}$, the friction force of the orbiting plate and fixed plate, as well as the gravity, inertia force and inertia moment used to balance the rotor system [3]. 
The geometric parameters of balance weights $m_{p 1}$ and $m_{p 2}$ are determined by the rotor system balance. The relationship between the mass of the crankshaft pin, the orbiting scroll assembly, and the balance weights is

$$
\left(m_{1}+m_{2}\right) l_{1}+m_{p 2} r_{p 2}=m_{p 1} r_{p 1}
$$

where $r_{p 1}$ is the distance between the centroid of counterweight $m_{p 1}$ and the rotation center; $r_{p 2}$ is the distance between the centroid of counterweight $m_{p 2}$ and the rotation center.

The orbiting scroll revolving translational motion characteristics shows that any point of the orbiting scroll is in the same movement rule. The direction of the frictional force is opposite to the orbiting scroll gravity (hinge $B$ ) moving, and the force role point is on the overturning moment action line [4]:

$$
\begin{aligned}
& F_{f d 1}=\mu_{d} F_{d 1}, \\
& F_{f d_{2}}=\mu_{d} F_{d 2} .
\end{aligned}
$$

$M_{\text {sd }}$, the friction torque on the orbiting scroll center (hinge $B$ ) caused by the friction forces $F_{f d 1}$ and $F_{f d 2}$, is as follows:

$$
M_{\mathrm{sd}}=\mu_{d} R_{t}\left(F_{d 1}-F_{d 2}\right) \sin (\eta-\gamma),
$$

where $\eta$ is the azimuth angle between the action line and the $x$-axis; $\gamma$ is the azimuth angle between the direction of $F_{f d}$ and the $x$-axis; $R_{t}$ is the radius of overturning moment action circle; $\mu_{d}$ is the friction coefficient of orbiting scroll and fixed scroll.

$F_{d 1}$ and $F_{d 2}$ can be obtained by the axial force balance of the orbiting scroll [5]:

$$
F_{d 1}-F_{d 2}=-\frac{M_{m}}{R_{t}}
$$

So, (12) can be rewritten as

$$
M_{\mathrm{sd}}=\mu_{d} M_{m} \sin (\gamma-\eta),
$$

where $M_{m}$ is the overturning moment acting on the bearing drive surface.

\section{Input Torque}

Excluding the influence of friction hinge deputy, the input torque, $T_{\mathrm{in}}$, can be considered to have three parts:

(1) $T_{d}$, inertia torque, to overcome inertia influence to maintain the driving rod uniform speed;

(2) $T_{w}$, gravitational torque, to balance the effect of gravity;

(3) $T_{F}$, external load torque, to balance the outside load.
For the scroll compressor, with uniform speed and conducted rotor system balance design, by the virtual work equation [6], input torque is as follows:

$$
\begin{gathered}
T_{d}=-\frac{J_{2} \ddot{\theta}_{2} \delta \theta_{2}}{\delta \theta_{1}}+\frac{J_{3} \ddot{\theta}_{3} \delta \theta_{3}}{\delta \theta_{1}}, \\
T_{w}=\frac{m_{3} g l_{3} \cos \theta_{3} \delta \theta_{3}}{\delta \theta_{1}}, \\
T_{F}=M_{t}-\frac{\left(M_{z}+M_{\mathrm{sd}}\right) \delta \theta_{2}}{\delta \theta_{1}},
\end{gathered}
$$

where $M_{t}=F_{t}\left(\theta_{1}\right) l_{1}$, which is the resistance moment caused by the tangential gas force acting on the crankshaft rotation center through the orbiting scroll.

$\delta \theta_{2}$ and $\delta \theta_{3}$ are given by the formulae (2) and (3) variational solution, respectively:

$$
\begin{gathered}
\delta \theta_{2}=\frac{l_{1}\left[l_{2} \sin \left(\theta_{2}-\theta_{1}\right)+l_{4} \sin \theta_{1}\right]}{l_{2}\left[l_{1} \sin \left(\theta_{2}-\theta_{1}\right)-l_{4} \sin \theta_{2}\right]} \delta \theta_{1}, \\
\delta \theta_{3}=\frac{l_{1}\left[l_{3} \sin \left(\theta_{3}-\theta_{1}\right)-l_{4} \sin \theta_{1}\right]}{l_{3}\left[l_{1} \sin \left(\theta_{3}-\theta_{1}\right)-l_{4} \sin \theta_{3}\right]} \delta \theta_{1} .
\end{gathered}
$$

The mini-crank quality $\left(m_{3}\right)$ could be negligible in the dynamical analysis, due to the structural characteristics of the scroll compressor. Thus, when the scroll compressor is in a uniform speed, the input torque can be expressed as

$$
T_{\text {in }}\left(\theta_{1}\right)=M_{t}-\frac{\left[M_{z}+J_{2} \ddot{\theta}_{2}+M_{\text {sd }}\right] \delta \theta_{2}}{\delta \theta_{1}} .
$$

When a scroll compressor is in ideal working condition, $\ddot{\theta}_{2}=0, \delta \theta_{2}=0$, the input torque is

$$
T_{\text {in }}\left(\theta_{1}\right)=M_{t} .
$$

Kinematics and kinetics show that the conditions of drive rod doing whole rotary motion [7] are

(1) the sum of the shortest and longest rod length should be less than or equal to the length of the remaining two;

(2) the shortest rod is connected frame bar.

The scroll compressor equivalent four-bar mechanism shows that regardless of the existence of rod size error, $l_{1}$ and $l_{4}$, rotation consisting deputy vice must be a complete rotation.

So,

$$
l_{1}+l_{2} \leq l_{3}+l_{4}
$$

or

$$
l_{1}+l_{4} \leq l_{3}+l_{2} .
$$

And when the actual length of $l_{3}$ is less than $l_{1}$, there must be clearances that exist in hinged deputy to ensure the drive rod doing whole rotary motion. 
TABLE 1: Parameters of the equivalent parallel four-bar mechanism.

\begin{tabular}{lc}
\hline Parameters & Values \\
\hline$l_{1}(\mathrm{~mm})$ & 4.5 \\
$l_{2}(\mathrm{~mm})$ & 115 \\
$l_{3}(\mathrm{~mm})$ & 4.5 \\
$l_{4}(\mathrm{~mm})$ & 115 \\
$J_{2}\left(\mathrm{Kg} \cdot \mathrm{m}^{2}\right)$ & 0.142 \\
\hline
\end{tabular}

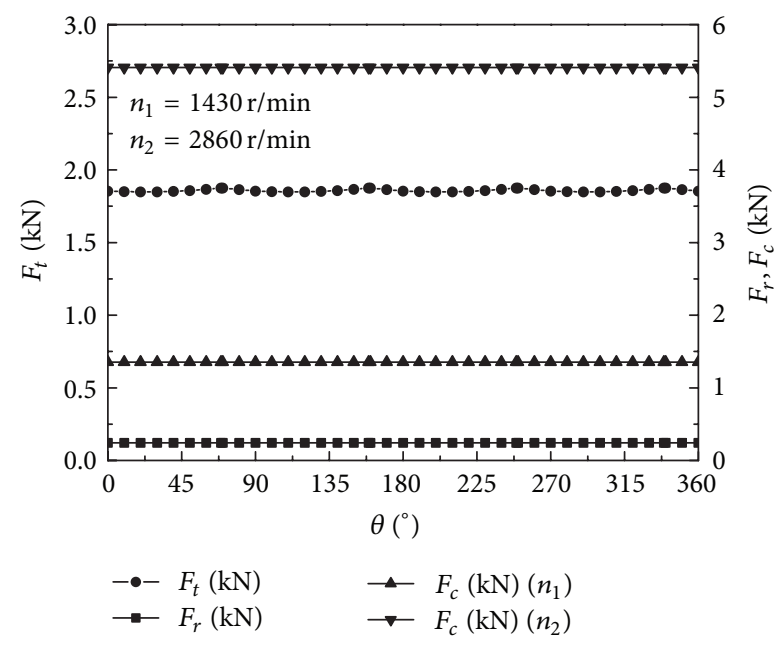

Figure 4: The diagram of external force.

\section{Model Verification}

Taking a twin-wraps scroll compressor as an example, we analyzed the effect of size error on input torque, without considering the mechanism clearances and hinge friction. Only take the shortest lever $l_{1}$ into account.

Table 1 shows the parameters of the equivalent fourbar mechanism parameters for input torque analysis. The external load changing with the crank angle of the examples of scroll compressor is shown in Figures 4 and 5; where radial gas force $F_{r}$ and orbiting scroll assembly inertial force $F_{c}$ are certain values at a certain speed, and the inertial force $F_{c}$ increases rapidly with the speed. The rotation moment $M_{Z}$ and the resistance moment $M_{t}$ change with the crank angle $\theta$ at the same discipline of tangential gas force $F_{t}$. The friction moment $M_{\text {sd }}$ increases with the speed quickly but is approximately constant at a certain speed.

It can be seen from Figures 6, 7, and 8:

(1) as the scroll compression is in ideal working condition, the variation of input torque of scroll compression is the same with the resistance moment $M_{t}$, as shown in Figure 6 with slight variation. So, the scroll compressor is designed generally without flywheel setting needed;

(2) when the equivalent parallel four linkage mechanism size error occurs, the actual input torque of compressor differs from the ideal condition a lot.

First, in the mini-crank reverse running area to the crankshaft, the input torque will deviate and strongly depend

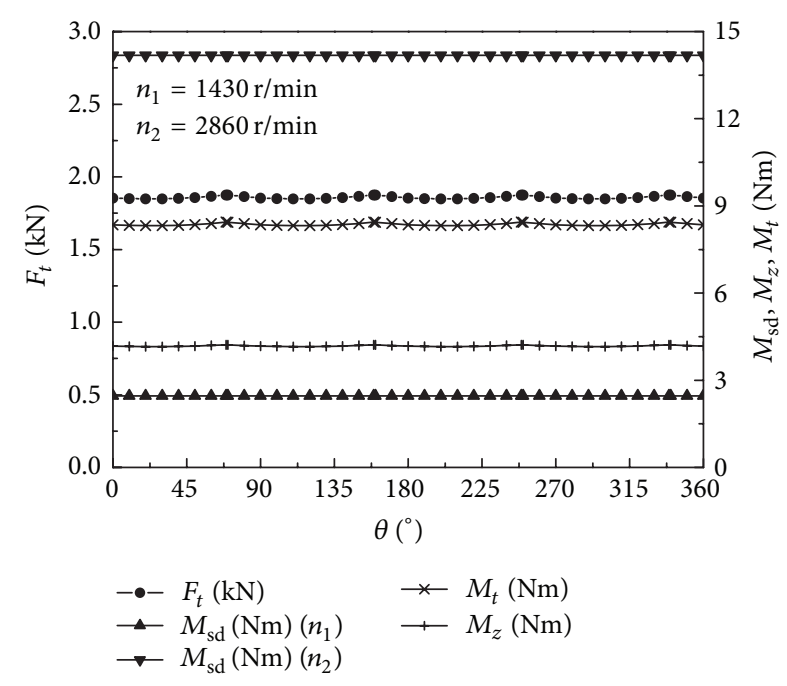

FIgURE 5: The diagram of external moment.

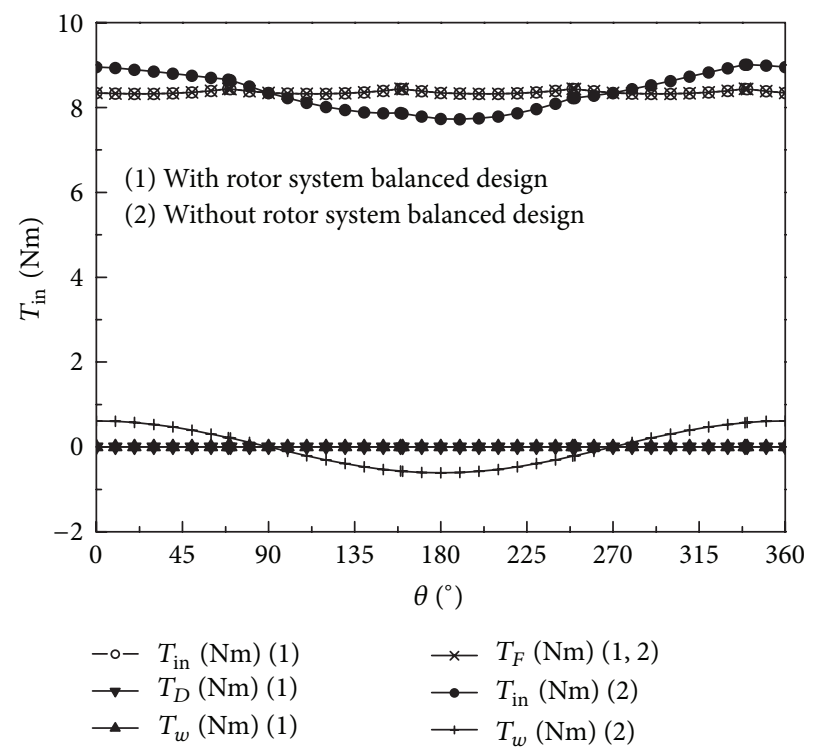

FIGURE 6: The diagram of input torque in ideal working.

on the size error and crankshaft speed. But, under different conditions, the value is insignificant and little change occurs in the amplitude in the region.

Second, the input torque will cause great fluctuation as mini-crank rotation direction changes. Comparison shows that the mini-crank changes in the same direction as the crankshaft, extreme fluctuations is the maximum and the higher the speed, the greater extremes.

Meanwhile, the scroll compressor structure determines that the more positive deviation $l_{2}$ (or negative deviation $l_{4}$ ), the more extreme fluctuations increase and the more negative deviation $l_{1}$ (or positive deviation $l_{3}$ ), the more extreme fluctuations reduces. 


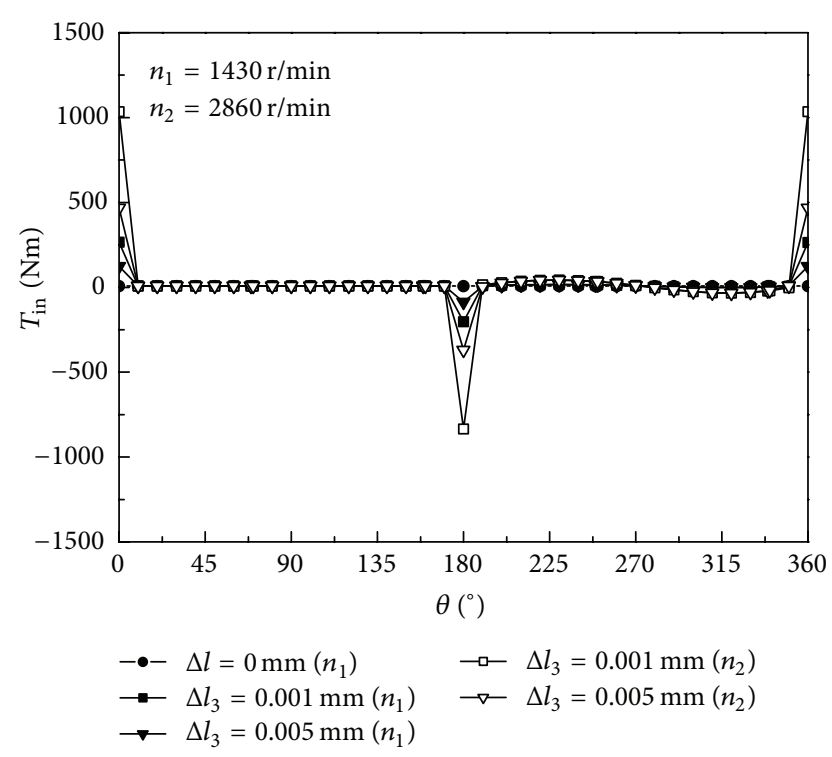

FIGURE 7: The diagram of the input torque.

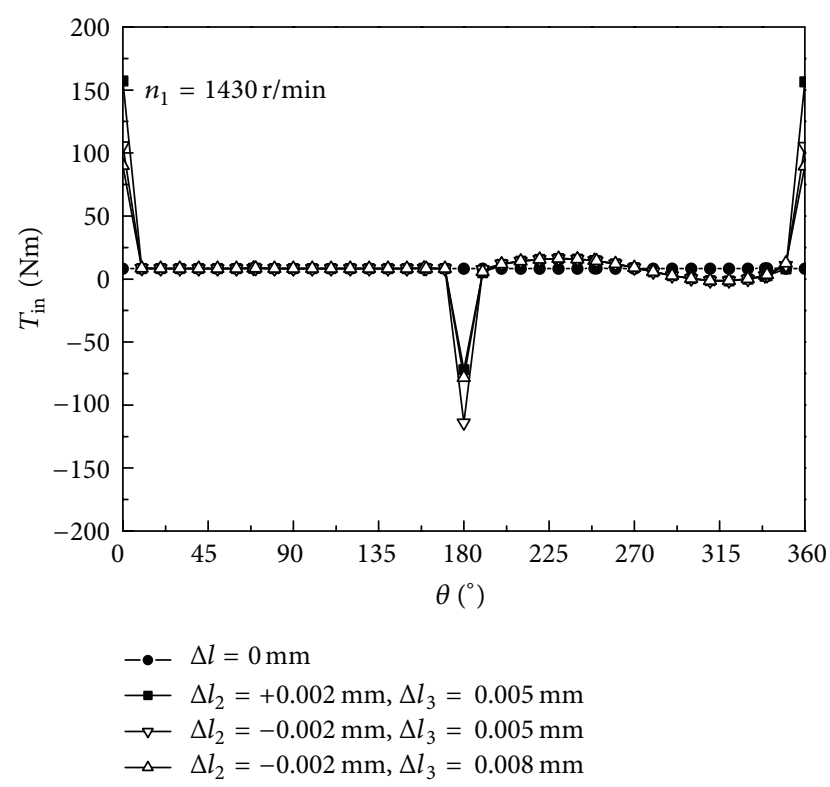

FIGURE 8: The comparison of input torque.

\section{Conclusions}

Based on the fundamental principle of plane linkage mechanism, effect of mechanism error on input torque of a completely dynamic balancing of scroll compressor was analyzed. Mechanism size error has a big influence on the drive rod and connecting rod collinear. The drive crankshaft eccentricity is beneficial for error reduction but causes more friction loss in the wrap of orbiting and fixed scroll. The appropriate precision should be selected, reducing the adverse effect caused by size error.

\section{Conflict of Interests}

The authors declare that there is no conflict of interests regarding the publication of this paper.

\section{Acknowledgment}

This work was supported by the National Natural Science Foundation of China (no. 51265026).

\section{References}

[1] Z. Liu, J. Wang, and J. Qiang, Scroll Fluid Machinery and Scroll Compressor, Machinery Industry Press, Beijing, China, 2009.

[2] C. Li, R. Zhao, and Z. Liu, "Effect of mechanism error of scroll compressor on sealing clearance," Lubrication Engineering, vol. 32, no. 7, pp. 66-68, 71, 2007.

[3] M. Zhao, S. Yu, C. Li, and Y. Yu, "The rotor system balance design of scroll fluid machinery," Applied Mechanics and Materials, vol. 160, pp. 268-272, 2012.

[4] M. Zhao, S. Yu, C. Li, and Y. Yu, "Analysis of orbiting scroll overturning for scroll compressor," Applied Mechanics and Materials, vol. 226-228, pp. 576-579, 2012.

[5] Z. Gu, Y. Yu, and S. Feng, Scroll Compressor and Other Scroll Machinery, Shanxi Science and Technology Press, Xi'an, China, 1998.

[6] J. Sun and C. Zhang, Structural Mechanics, Chongqing University Press, 2001.

[7] H. Sun, Mechanical Principles, Higher Education Press, Beijing, China, 2001. 

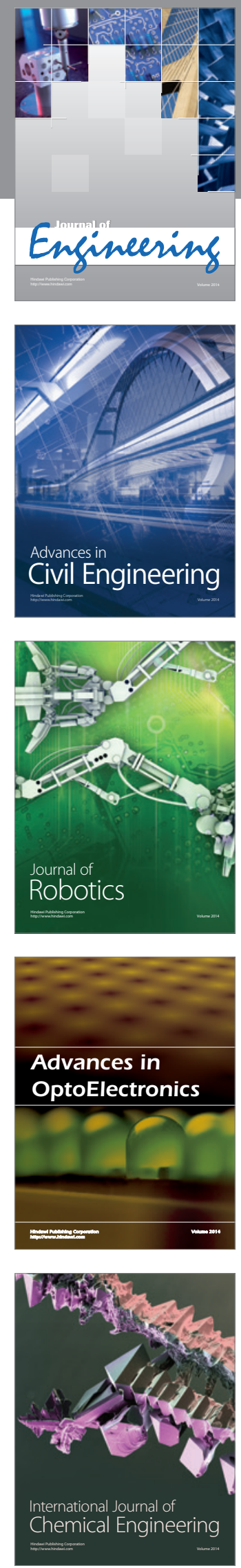

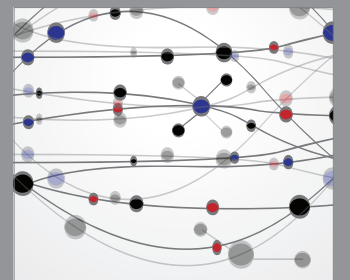

The Scientific World Journal
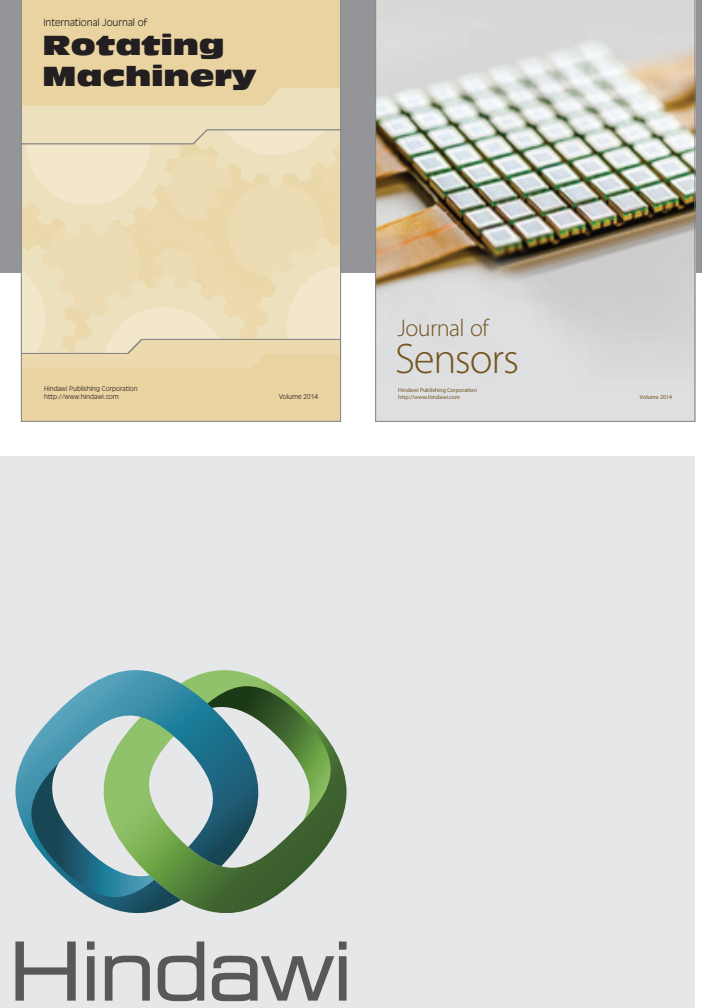

Submit your manuscripts at http://www.hindawi.com
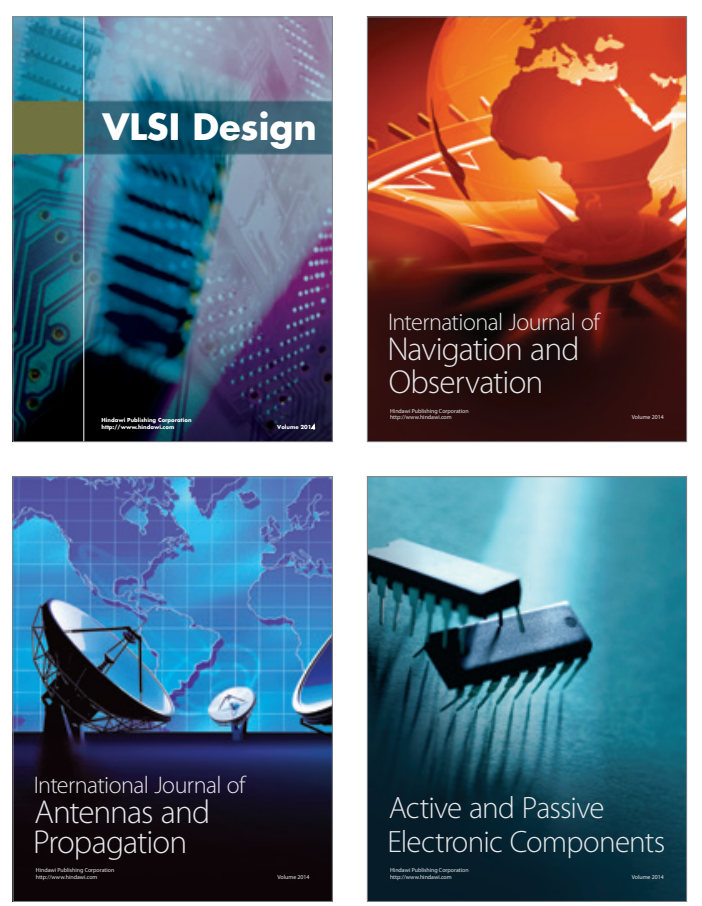
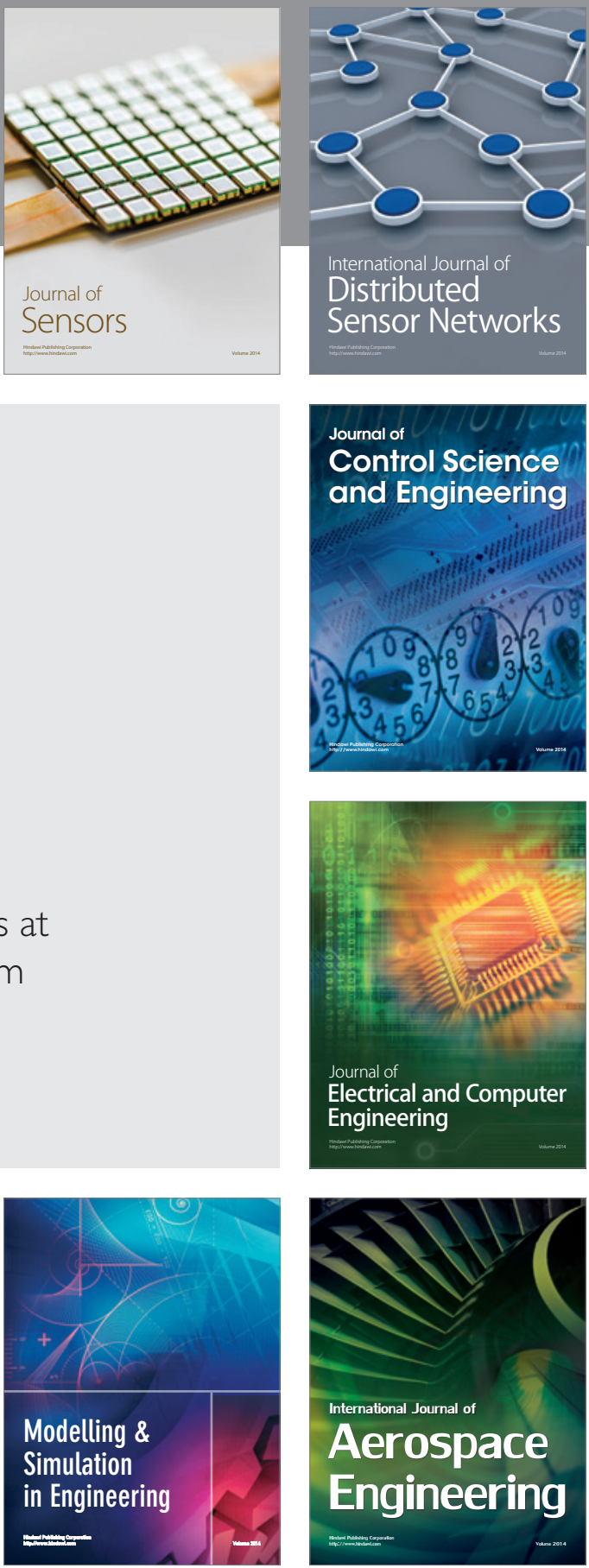

Journal of

Control Science

and Engineering
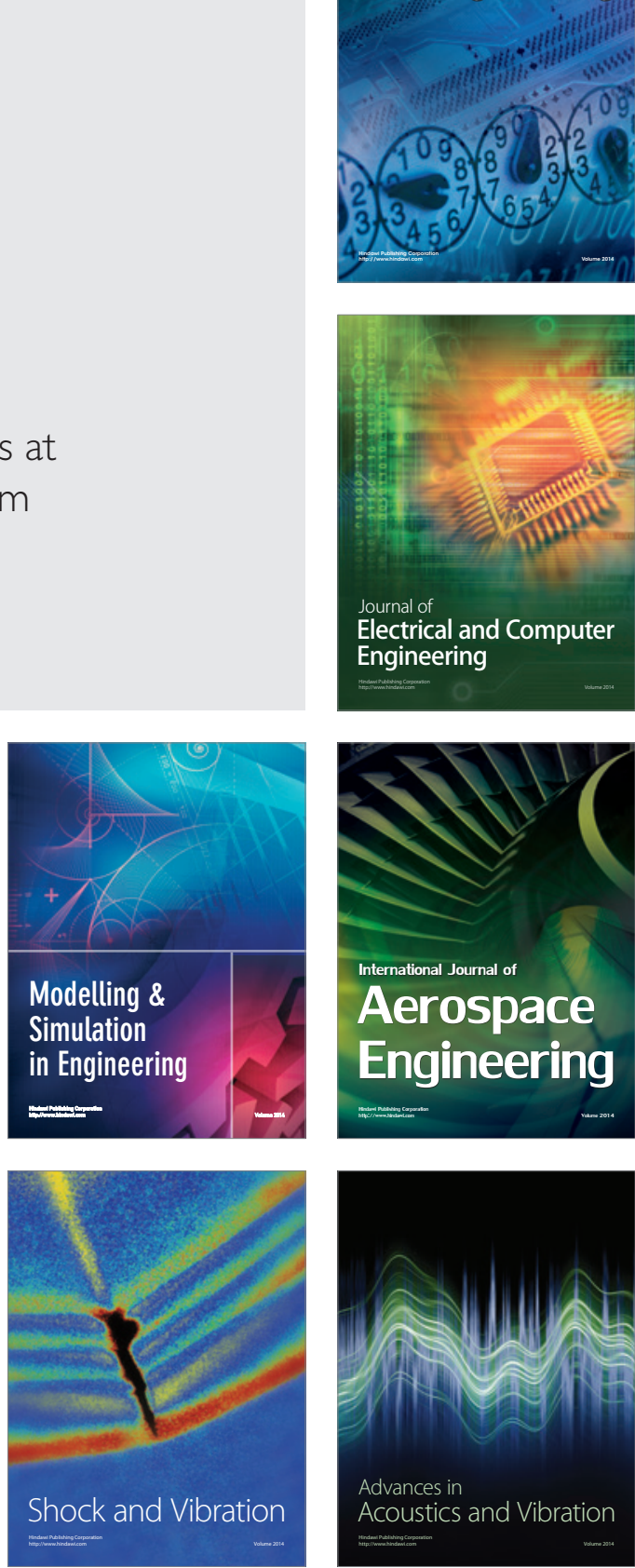